\title{
Analysis of temporal-longitudinal- latitudinal characteristics in the global ionosphere based on tensor rank-1 decomposition
}

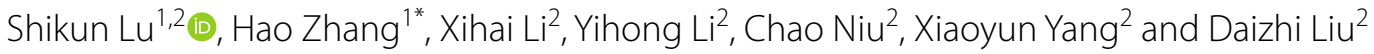

\begin{abstract}
Combining analyses of spatial and temporal characteristics of the ionosphere is of great significance for scientific research and engineering applications. Tensor decomposition is performed to explore the temporal-longitudinallatitudinal characteristics in the ionosphere. Three-dimensional tensors are established based on the time series of ionospheric vertical total electron content maps obtained from the Centre for Orbit Determination in Europe. To obtain large-scale characteristics of the ionosphere, rank-1 decomposition is used to obtain $U^{(1)}, U^{(2)}$, and $U^{(3)}$, which are the resulting vectors for the time, longitude, and latitude modes, respectively. Our initial finding is that the correspondence between the frequency spectrum of $U^{(1)}$ and solar variation indicates that rank-1 decomposition primarily describes large-scale temporal variations in the global ionosphere caused by the Sun. Furthermore, the time lags between the maxima of the ionospheric $U^{(2)}$ and solar irradiation range from 1 to $3.7 \mathrm{~h}$ without seasonal dependence. The differences in time lags may indicate different interactions between processes in the magnetosphere-ionosphere-thermosphere system. Based on the dataset displayed in the geomagnetic coordinates, the position of the barycenter of $U^{(3)}$ provides evidence for north-south asymmetry (NSA) in the large-scale ionospheric variations. The daily variation in such asymmetry indicates the influences of solar ionization. The diurnal geomagnetic coordinate variations in $U^{(3)}$ show that the large-scale EIA (equatorial ionization anomaly) variations during the day and night have similar characteristics. Considering the influences of geomagnetic disturbance on ionospheric behavior, we select the geomagnetic quiet GIMs to construct the ionospheric tensor. The results indicate that the geomagnetic disturbances have little effect on large-scale ionospheric characteristics.
\end{abstract}

Keywords: Large-scale ionospheric characteristics, Tensor rank-1 decomposition, Time lags, Geomagnetic northsouth asymmetry

\section{Introduction}

Known as the 'space weather display', the ionosphere is an important part of the Sun-Earth system. Ionospheric characteristics can reflect both variations in the solarterrestrial environment and solid Earth and atmosphere (Afraimovich et al. 2006; Yoshikawa et al. 1999). With advances in information technology, radio waves have

\footnotetext{
*Correspondence: haozhang@mail.tsinghua.edu.cn

1 Department of Electronic Engineering, Tsinghua University, Beijing 100084, China

Full list of author information is available at the end of the article
}

become a significant carrier for information. Due to the ionospheric influence on radio wave propagation, the impact of the ionosphere on people's lives is increasing. Both national security, e.g., space-borne synthetic-aperture radar system (Tsynkov 2009) and over-the-horizon radar (Hu et al. 2016), and people's lives, e.g., GPS position and high-voltage networks (Liu et al. 2013), are closely related to the ionosphere. As a result, ionospheric research is of great value for both theory and application.

Extensive research has been conducted regarding ionospheric spatial and temporal characteristics. With the help of the space technology, especially GPS, researchers 
have gained insight into the shape and behavior of the ionosphere. However, conventional research usually focuses on temporal and spatial variations separately. Ren et al. (2008) examined the electron temperature and total ion density in the sunset equatorial topside ionosphere and found clear seasonal dependence and significant anti-correlation using the longitudinal variations in ionosphere. Mu et al. (2010) analyzed the correlation between ionospheric longitudinal harmonic components (WN3 and WN4) and upper atmospheric tides. They found that atmospheric tidal DE2 and DE3 patterns were important factors in the ionospheric WN3 and WN4 structures. Kil and Paxton (2011) reviewed the causal link between longitudinal plasma density structure, vertical plasma drifts, and atmospheric tides. These previous studies only focused on longitudinal variations. Xu et al. (2013) investigated the influence of declination and thermospheric wind on longitude differences in midlatitude ionospheric total electron content (TEC) by analyzing the data distributed on both sides of the longitudes with zero declination. Wang et al. (2015) and Wang and Zhang (2017) studied the longitudinal and diurnal differences in midlatitudinal electron density from a global perspective using the global ionosphere-thermosphere model. They quantified the effects from in-situ physical processes and atmospheric tides from the low atmosphere. These researchers studied spatial variations in the ionosphere, ignoring temporal variations by fixing or removing them. Other studies have only focused on the temporal variation of the ionosphere, such as the seasonal and diurnal changes (Liu et al. 2009; Lee et al. 2011; Coyne and Belrose 1972; Wright 1962). Roughly compressing the data in one certain dimension results in loss of information in other dimensions or coupling between dimensions. Identical to matrix analysis, compared to eigenvectors obtained from the singular value decomposition, one column or row contains much less information than the matrix.

Some research has been made to jointly analyze temporal and spatial variations. Wen et al. (2007) investigated ionospheric temporal and spatial variations during the August 18, 2003, storm over China to reveal ionospheric storm evolution processes using time series ionospheric electron density maps. Yao et al. (2013) conducted research on temporal and spatial variations in ionospheric electron density profiles over South Africa during strong geomagnetic storms using the 3D structure of ionospheric electron density and its response to geomagnetic storms. Essentially, both research groups tried to analyze the spatiotemporal variations with a time series of spatial variation results. However, spatial and temporal variations of the ionosphere are correlated (Talaat and Zhu 2016). Therefore, this strategy cannot provide deep insight into spatiotemporal variations in the ionosphere. By formulating statistics using separate bins, AraujoPradere et al. (2005) determined that ionospheric variability for quiet and disturbed conditions is a function of local time, geomagnetic activity, season, and geomagnetic latitude. In their research, the data were divided into different bins that disregarded the coupling between factors. For example, for seasonal dependence, the data were simply divided into five seasonal bins, regardless of spatiotemporal coupling. Afraimovich et al. (2008) found that Global Electron Content (GEC) well reflected Sun dynamics. However, GEC is calculated by the summation of the global TEC, removing spatial information. Lean et al. (2016) studied the distinctive spatial patterns of TEC response to solar, seasonal, diurnal, and geomagnetic influences using a new statistical model based on the linear summation of different influences, but this also neglects the coupling between them. Consequently, the strategy, separately analyzing or roughly compressing the spatial and temporal characteristics, may destroy the spatiotemporal coupling. However, research has been conducted to address this issue. Chen et al. (2015) illustrated the importance of different types of spatial-temporal variations to the TEC over North America using the empirical orthogonal function (EOF). Talaat and Zhu (2016) analyzed the spatial and temporal variation of total electron content revealed by principal component analysis (PCA). Although these studies can separate spatial and temporal variations, they cannot separately provide detailed spatial information on variations in longitude and latitude. Generally, previous research on ionospheric spatiotemporal variations has not decoupled correlations in time, longitude, and latitude and has not truly combined analyses of these three modes simultaneously.

Our dataset, a time series of global ionospheric maps, constitutes a data cube, represented by a third-order tensor. As an efficient method to analyze multidimensional arrays, tensor rank decomposition can be used to address multilinear algebra problems, e.g., combined analyses of time, longitude, and latitude, which would be intractable for classical techniques (Grasedyck et al. 2013). In addition, similar to the widely used methods for spatiotemporal analysis, PCA and EOF, tensor rank decomposition can also analyze different scaled features in data tensors (Filisbino et al. 2013). Notably, rank-1 decompositions can extract large-scale characteristics of time, longitude, and latitude simultaneously. In this study, we utilize GPS-derived VTEC data supplied by CODE from 2011 to 2013. We combine an in-depth investigation on the global spatial features with temporal variations in the ionosphere based on tensor rank-1 decomposition. "Description of data and methods" section provides a description of the data information and methods. The 
resulting ionospheric temporal-longitudinal-latitudinal characteristics are presented in "Results and discussion" section, followed by conclusions in "Conclusions" section.

\section{Description of data and methods VTEC data source}

Carrying abundant information about climatological variations of the ionosphere (Ercha et al. 2015), VTEC has been extensively used to investigate the ionosphere. CODE, as one of the IGS (International Global Navigation Satellite System Service) laboratories, can supply global VTEC data with 2-h time resolution (Wei et al. 2009). The laboratory uses dual-frequency code and phase GPS data from more than 200 stations worldwide (Hernández-Pajares et al. 2009; Rao and Dutt 2014). In the current research, a global ionospheric map (GIM) is the result of VTEC data derived from CODE. The selected dataset is named after CODE (ftp://ftp.unibe. ch/aiub/CODE). The VTEC in CODE is modeled using a spherical harmonics expansion up to order 15 . Simultaneously, 3328 parameters are used to represent the global VTEC distribution. As a result, the CODE dataset can provide abundant detailed ionospheric variations in both space and time.

The spatial extent of GIM ranges from $180^{\circ} \mathrm{W}$ to $180^{\circ}$ $\mathrm{E}$ in longitude and from $87.5^{\circ} \mathrm{S}$ to $87.5^{\circ} \mathrm{N}$ in latitude. The size of a GIM cell is $5^{\circ}$ in longitude and $2.5^{\circ}$ in latitude. The time-varying GIMs for VTEC, 2D spatial maps, can constitute a third-order tensor by the arrangement with time.

\section{Tensor rank decomposition}

The widespread application of multi-sensor technology and the emergence of high-dimensional datasets have highlighted the limitations of traditional flat-view matrix methods and shown the necessity of moving toward more versatile tools. High-order statistics for multivariate stochastic variables promote the development of multilinear algebra, which is the algebra of higher-order tensors. The range of applications for tensor techniques has expanded widely in signal processing and data analysis, e.g., computational tasks in electronic structure calculations and solution to stochastic and parametric partial differential equations (Grasedyck et al. 2013; Khoromskaia and Khoromskij 2014; Espig et al. 2014).

As a function of geographic coordinates, geomagnetic latitude, and local time, the VTEC is highly variable in time, longitude, and latitude. To jointly analyze timelongitude-latitude characteristics in the ionosphere, we propose tensor rank decomposition. Moreover, in this research, we mainly use rank-1 decomposition, i.e., the best rank-1 approximation, to analyze large-scale characteristics in the ionosphere. Rank-related issues in multilinear algebra are thoroughly different from their matrix counterparts. The existing framework for vector and matrix algebra appears to be insufficient and/or inappropriate for high-dimensional dataset. The tensors, which are higher-order equivalents of vectors (first order) and matrices (second order), are also known as multidimensional arrays. Considering our application, the dataset here constitutes a three-mode tensor, which can be denoted as $\mathscr{A}_{T \times \text { lon } \times \text { lat. }}$. The three modes are universal time (UT), geographic longitude, and latitude, respectively. Therefore, $\mathscr{A}_{T \times \text { lon } \times \text { lat }}$ is affected by solar irradiation, thermospheric neutral winds, magnetosphere, and other factors. Subsequently, the dataset can be written as a function of those factors, $\mathscr{A}_{T \times \text { lon } \times \text { lat }}\left(e_{1}, e_{2}, e_{3}, \ldots\right)$ , where $\left(e_{1}, e_{2}, e_{3}, \ldots\right)$ are the respective sources of the global ionospheric variability. These factors are expected to cause ionospheric variability. Here, we use $\mathscr{A}$ to represent $\mathscr{A}_{T \times \text { lon } \times \text { lat }}\left(e_{1}, e_{2}, e_{3}, \ldots\right)$ for simplicity. The tensor rank decomposition can be formulated as follows:

$$
\begin{aligned}
\hat{\mathscr{A}} & =\arg _{\hat{\mathscr{A}}} \min \|\mathscr{A}-\hat{\mathscr{A}}\|_{F}, \\
\text { s.t. } \hat{\mathscr{A}} & =\sum_{k=1}^{r} \lambda_{k} U_{k}^{(1)} \circ U_{k}^{(2)} \circ U_{i}^{(3)}, \\
\left\|U_{k}^{(n)}\right\| & =1,(n=1,2,3 ; k=1,2, \ldots, r) .
\end{aligned}
$$

where $U_{k}^{(1)}, U_{k}^{(2)}$, and $U_{k}^{(3)}$ are the resulting unit-form vectors on time, longitude, and latitude modes, respectively. $U_{k}^{(1)}, U_{k}^{(2)}$, and $U_{k}^{(3)}$ are also affected by solar irradiation, thermospheric neutral winds, magnetosphere, and other factors. Then, $U_{k}^{(n)}$ is the abbreviation of $U_{k}^{(n)}\left(e_{1}, e_{2}, e_{3}, \ldots\right)$. As described previously, $\left(e_{1}, e_{2}, e_{3}, \ldots\right)$ are the sources of ionospheric variability. $\lambda_{k}$ is the amplitude. The vectors are defined as $U_{k}^{(n)}:=\left(u_{k 1}^{(n)}, \ldots, u_{k i}^{(n)}, \ldots, u_{k N}^{(n)}\right)$, and $N$ is the length of tensor in $n$th mode. $\hat{\mathscr{A}}$, whose rank is $r$, is the approximation of $\mathscr{A}$, and its component $\hat{a}_{i_{1} i_{2} i_{3}}$ is calculated using the sum of the outer product 'o', resulting in $\hat{a}_{i_{1} i_{2} i_{3}}=\sum_{k=1}^{r} u_{k i_{1}}^{(1)} u_{k i_{2}}^{(2)} u_{k i_{3}}^{(3)} \cdot\|\cdot\|_{F}$ is the Frobenius norm. Essentially, this decomposition tries to find a rank-r tensor to approximate the data tensor, based on the condition that the Frobenius norm of error is as small as possible. Thus, rank- $r$ decomposition extracts the first $r$ principal components of the tensor. This decomposition has been applied in computer vision, graph analysis, and neuroscience (Lu et al. 2006; Kolda and Bader 2009). Based on this explanation, the tensor decomposition can jointly analyze the characteristics of each dimension in the dataset. This constrained optimization problem can be analyzed using the Lagrange multipliers technique (Lathauwer et al. 2000). 
To describe the method clearly, we show the results for $r=1,5,10,20$, and 30 in Fig. 1. From those subfigures, we find that, with increasing $r$, the results include more and more details of ionospheric variation. The first few components capture smooth or large-scale structures of the ionosphere, as shown in Fig. 1 for $r=1$. In our application, to analyze large-scale ionospheric structures, we only focus on the first principal component of the timelongitude-latitude characteristics, where $r=1$. Because we mainly focus on the large-scale characteristics of the ionosphere, the details in the results are not necessarily expected to show close correspondence with the original data. Furthermore, the two crests in the subplots, ' $r=1$ CODG-1' and ' $r=1$ CODG-2' in Fig. 1, indicate the variation caused by the Sun and geomagnetic field (Franke et al. 2003). Such phenomenon is called the ionospheric fountain effect, which plays an essential role in the equatorial ionization anomaly (EIA). This anomaly is especially significant during daytime, because of the ionizing effect of solar radiation. Thermospheric winds in the equatorial $E$ region drag particles across the geomagnetic field lines $\mathbf{B}$, creating an eastward electric field $\mathbf{E}$. Due to $\mathbf{E} \times \mathbf{B}$ forces and the influence of gravity and pressure gradient forces, particles travel along field lines to higher latitudes (Hanson and Moffett 1966). Thus, there is one crest about the VTEC appearing on each side of the equator.

\section{Results and discussion}

As described in the methods description subsection, rank-1 decomposition generates three vectors, $U^{(1)}, U^{(2)}$, and $U^{(3)}$ (the subscripts for $U_{k}^{(n)}$ are omitted). By equation (1), the best rank-1 approximation $\hat{\mathscr{A}}$ can be obtained. The subfigures in Fig. 1, ' $r=1$ CODG-1/2', show the best rank-1 approximation for the CODG dataset (14:0016:00 UT on January 1, 2011). From the contrast between the original and rank-1 subplots in Fig. 1, the approximation clearly extracts the components for the large scale. Based on the results of tensor rank-1 decomposition, we analyze these three vectors, $U^{(1)}, U^{(2)}$ and $U^{(3)}$ to obtain the large-scale characteristics in the ionosphere with regard to time, longitude, and latitude.

\section{Fourier spectral analysis of $U^{(1)}$}

Because solar energy ionizes atmospheric molecules and creates the ionosphere, the ionospheric VTEC tensor is largely controlled by the Sun (Goldberg 1966; Schunk and Nagy 2009). Even though the temporal variation in the ionosphere is also affected by variations in geomagnetic field, magnetosphere, and atmosphere excluding solar irradiation, for the timescale of this research, the temporal variations in geomagnetic field, magnetosphere, and atmosphere are derived from solar irradiation as well. Thus, as demonstrated by Afraimovich et al. (2008), the global, long-timescale characteristics of the ionosphere are determined by solar irradiation. The rank-1 decomposition primarily extracts the long periodic

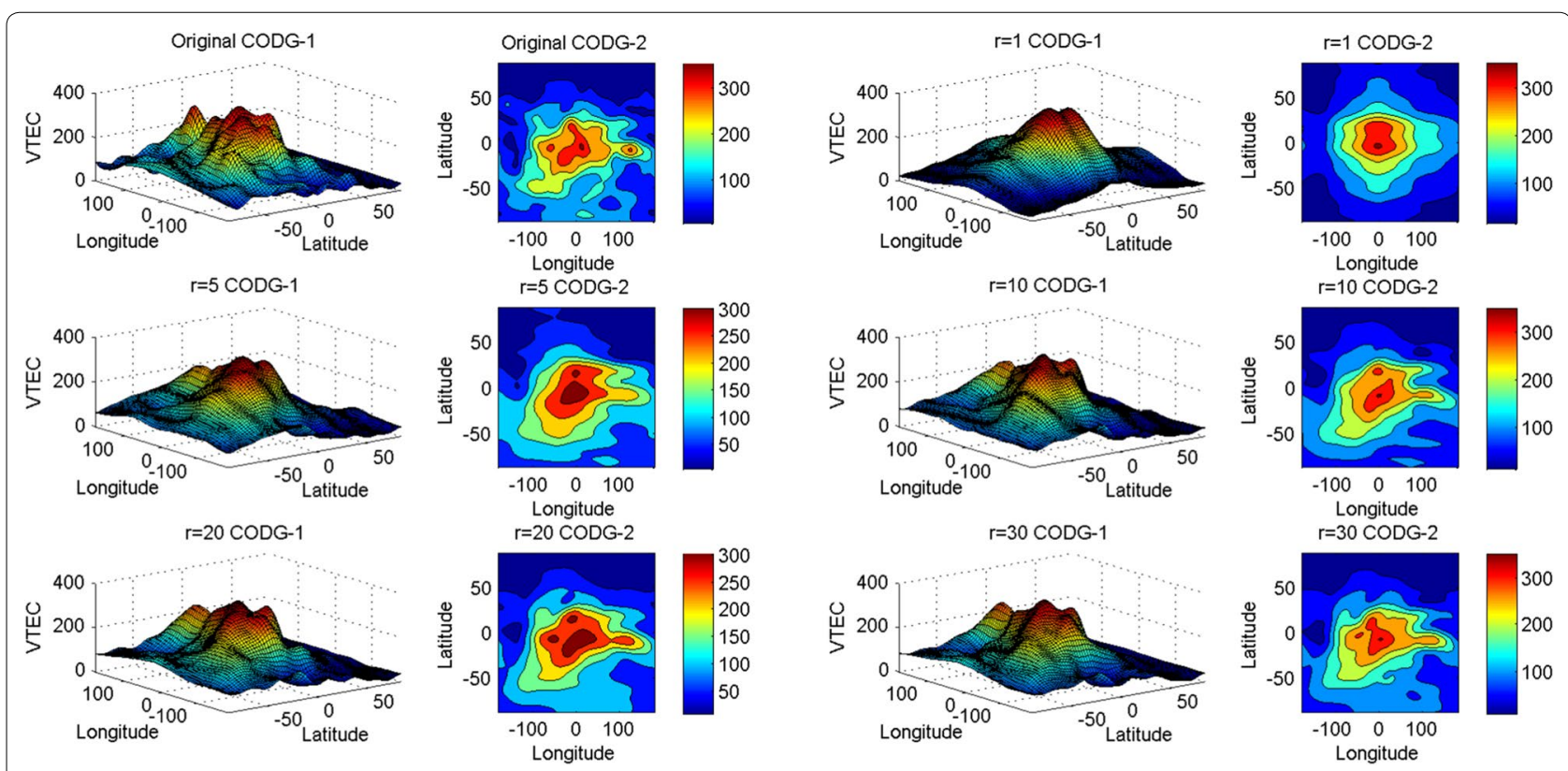

Fig. 1 The original ionospheric VTEC map for 14:00 - 16:00 UT on January 1, 2011 and its corresponding tensor rank decomposition, $r=1,5,10$, 20 , and 30 . The labels, '- $1^{\prime}$ and ' $-22^{\prime}$, represent the surface and contour plots, respectively. The geographic longitude ranges from $-180^{\circ}$ to $180^{\circ}$. The geographic latitude ranges from $-87.5^{\circ}$ to $87.5^{\circ}$. The units of VTEC are in $0.1 \mathrm{TECU}$ 


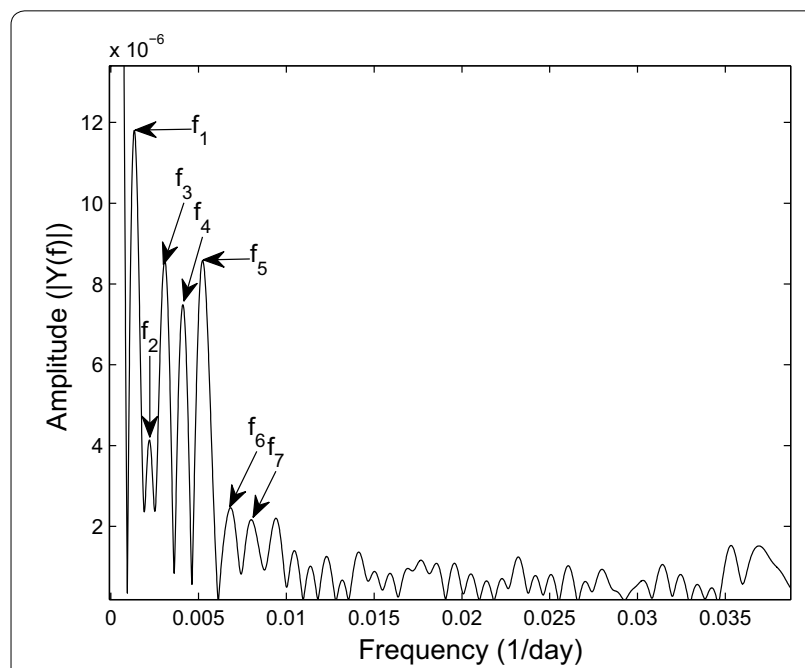

Fig. 2 The frequency spectrum for $U^{(1)}$ and the first seven main frequency components

variations in the ionosphere. Therefore, we hypothesize that the results of rank-1 decomposition for the time mode describe the ionospheric temporal variation mainly determined by solar irradiation.

As the global VTEC supplied by CODE is given with a 2 -h time resolution, one day is divided into 12 time intervals, which are UT1:(00:00-02:00),..,UT12:(22:00-24:00). We construct a 3D tensor using only one VTEC map each day from 2011 to 2013. As a result, we can achieve twelve tensors based on twelve UT intervals, and obtain twelve $U^{(1)}$. Because the frequency spectra of the twelve $U^{(1)}$ are almost identical, we arbitrarily select one $U^{(1)}$ to analyze. The tensor, constructed by the global VTEC map at UT8 every day from 2011 to 2013, is selected. The frequency spectrum of $U^{(1)}$ is shown in Fig. 2. We select the first seven primary frequency components, $f_{1}, \ldots, f_{7}$ . The frequency spectrum and its corresponding solar

Table 1 The first seven primary frequency components of $U^{(1)}$ and corresponding periodicities (in year) of the Sun (Polygiannakis et al. 2003) and Mg II Index (Hocke 2008)

\begin{tabular}{lllll}
\hline $\begin{array}{l}\text { Frequency } \\
\text { label }\end{array}$ & $\begin{array}{l}\text { Frequency } \\
\left(\mathbf{1 0 ^ { - 3 }} \mathbf{1 / \text { day) }}\right)\end{array}$ & $\begin{array}{l}\text { Periodicity } \\
\text { (year) }\end{array}$ & $\begin{array}{l}\text { Observed } \\
\text { solar perio-- } \\
\text { dicity (year) }\end{array}$ & $\begin{array}{l}\text { Periodic- } \\
\text { ity in Mg II } \\
\text { index (year) }\end{array}$ \\
\hline$f_{1}$ & 1.33 & 2.06 & $2.1 \pm 0.1$ & - \\
$f_{2}$ & 2.2 & 1.25 & $1.4 \pm 0.1$ & - \\
$f_{\Delta}$ & - & - & $1.07 \pm 0.08$ & - \\
$f_{3}$ & 3.08 & 0.90 & $0.97 \pm 0.06$ & 1 \\
$f_{4}$ & 4.11 & 0.67 & $0.67 \pm 0.05$ & - \\
$f_{5}$ & 5.23 & 0.52 & $0.49 \pm 0.04$ & 0.5 \\
$f_{6}$ & 6.82 & 0.40 & $0.42 \pm 0.04$ & - \\
$f_{7}$ & 8.0 & 0.34 & $0.34 \pm 0.02$ & - \\
\hline
\end{tabular}

periodicities are shown in Table 1 , where $T=1 / f$. There are various periodic variations in the ionosphere, including 27-day, daily, and yearly variations. Those periodic variations, e.g., 27-day and daily variations, are shortterm compared to 3 years. The tensor rank-1 decomposition mainly focuses on the large-scale structures. Thus, only long periodic variations are presented in Table 1 . In contrast, short-periodic variations will appear in the higher-rank decomposition.

As the nearest star, the Sun has an important effect on climate change and daily life on Earth. Hence, the periodicities of solar variation have been widely studied. There are various types of periodic solar activities. Significant cycles range from seconds to years (Atac et al. 2005), including the 1.3-year periodicity in solar rotation, which forms solar convection (Krivova and Solanki 2002; Guo et al. 2015; Moussas et al. 2005), 2-year periodicity (quasibiennial oscillation), and others, such as $0.9,0.67,0.53$, 0.4 , and 0.35-year (Polygiannakis et al. 2003). Furthermore, the formation of the ionosphere is primarily due to the ionization of the upper atmosphere by solar radiation. Therefore, temporal variations in the global ionosphere depends dominantly on the Sun. Table 1 shows a good corresponding relationship between the frequency spectrum of $U^{(1)}$ and solar irradiation. $f_{\Delta}$ corresponds to the 1.07-year period, which is missing in our results. This is probably due to the narrow interval between $f_{\Delta}$ and $f_{3}$, whose corresponding periods are $(1.07 \pm 0.08)$ year and $(0.97 \pm 0.06)$ year. Furthermore, based on Fourier Transform theory, the frequency resolution is $1 / T_{w}$, where $T_{w}$ is the length of the series. The frequency uncertainty is $\frac{1}{3 \mathrm{yr}}$ in our research. To resolve the periodicity, $(1.07 \pm 0.08)$ year, the length of the time series should be 10.4 year at least, because the frequency resolution should be larger than $0.096 \frac{1}{\mathrm{yr}}$, which is calculated by $\left(\frac{1}{f_{3}}-\frac{1}{f_{\Delta}}\right)$. Such a corresponding relationship among those periods indicates that the global ionospheric temporal variations are primarily influenced by the Sun.

The ionosphere is generalized by ultraviolet, $\mathrm{X}$-ray, and shorter wavelengths of solar irradiation. Extreme ultraviolet (EUV) is electromagnetic radiation, which has an important effect on the generation of the $\mathrm{F}$ region. As a proxy for solar EUV, the Mg II index has spectral component that include 1-year and 0.5-year periods (Hocke 2008), which are also shown in Table 1 . Therefore, the time mode result, $U^{(1)}$, has provided some information on solar EUV. However, some other periods disappeared, which may be due to the Mg II index merely being a proxy for EUV, rather than the whole wavelength of solar irradiation. Meanwhile, because rank-1 decomposition mainly extracts the large-scale structure, rapid variations in Mg II index may be shown in the high-order decomposition. The results only show temporal variation patterns 
Table 2 The longitude position of the maxima of $U^{(2)}$ and solar irradiation and longitude differences in each UT interval

\begin{tabular}{llll}
\hline Time interval & $\begin{array}{l}\boldsymbol{U}^{(\mathbf{( 2 )}} \text { Maximum } \\
\left(/^{\circ}\right)\end{array}$ & $\begin{array}{l}\text { Solar irradiation } \\
\text { maximum }\left(/^{\circ}\right)\end{array}$ & $\begin{array}{l}\text { Longitude } \\
\text { difference }\left(/^{\circ}\right)\end{array}$ \\
\hline UT1 & -150 & -180 & 30 \\
UT2 & -175 & 150 & 35 \\
UT3 & 145 & 120 & 25 \\
UT4 & 115 & 90 & 25 \\
UT5 & 90 & 60 & 30 \\
UT6 & 65 & 30 & 35 \\
UT7 & 25 & 0 & 25 \\
UT8 & -5 & -30 & 25 \\
UT9 & -25 & -60 & 35 \\
UT10 & -35 & -90 & 55 \\
UT11 & -105 & -120 & 15 \\
UT12 & -115 & -150 & 35 \\
\hline
\end{tabular}

in the ionosphere. The spatial variation, which is affected by the geomagnetic field and magnetosphere, is shown in the longitude and latitude mode analysis.

\section{Longitudinal patterns via $U^{(2)}$}

Solar irradiation is one of the primary drivers of ionospheric variability, especially those of large scale, as the ionosphere is created by solar irradiation through ionization. Thus, if the ionospheric variations are only affected by the Sun, the VTEC amplitude would be proportional to solar irradiation. Generally, VTEC positively correlates with solar irradiation. Rama et al. (2016) found that the solar irradiation reached a maximum between 12:0013:00 local time (LT). We mainly extract the longitude positions of the maxima of solar irradiation and ionospheric $U^{(2)}$ in the 12 UT intervals.

Similar to the acquisition of $U^{(1)}$, through rank-1 decomposition, $12 U^{(2)}$ can be achieved. Based on the conclusions in Reference Rama et al. (2016) and the 2-h time resolution, we believe the maximum of the solar irradiation on Earth is 12:00-14:00 LT. Then, we identify their central longitude position. The results are shown in Table 2 and Fig. 3. The $12 U^{(2)}$ represent the longitudinal wave- 1 structure, i.e., single peak and trough. Because the longitude corresponds to local time, the peaks and troughs indicate variations during day and night times. The longitude differences between the positions of the maxima of $U^{(2)}$ and solar irradiation are shown in Fig. 3 and Table 2.

From the differences between the red and black dotted lines in Fig. 3, we find that there are longitude lags between the maxima of $U^{(2)}$ and solar irradiation. As is known, for a $15^{\circ}$ longitude change, a one-hour time change is required. As shown in Fig. 3 and Table 2, the longitude difference primarily ranges from $25^{\circ}$ to $35^{\circ}$, except $55^{\circ}$ at UT10 and $15^{\circ}$ at UT11. To reduce the impact of those two outliers, $55^{\circ}$ and $15^{\circ}$ are removed to obtain a more robust average. The mean of the remaining longitude differences is $30^{\circ}$; the corresponding time lag is $2 \mathrm{~h}$.

Furthermore, we investigate the monthly variation in time lags between large-scale variations in ionosphere and solar irradiation. In each UT interval, using 1-month global ionospheric maps (GIMs) instead of 3-years, we can construct a tensor, $\mathscr{A}_{30 \times 73 \times 71}$, each month, where 30 indicates 30 days in a month. There will be 12 tensors per year for each UT interval, accordingly. There are 3 years of data from 2011 to 2013 (36 months in total). We obtain $36 \times 12$ tensors, where 36 is the number of months and 12 is the number of UT intervals. Therefore, for the 12 months from January to December, each month will have 36 samples, including 3 samples for each 12 UT range. The longitude positions of the maxima of $U^{(2)}$ and solar irradiation are used to investigate seasonal variations in the time lags. Based on the longitude differences between the maximum $U^{(2)}$ and solar irradiation, we calculate the time lags in each UT interval for the 12 months from January to December. As a result, 36 time lags can be obtained each month. We calculate the averages and standard deviations of the time lags, with the results shown in Fig. 4.

In Fig. 4, the average time lags exhibit substantial seasonal differences. The Lloyd seasons, D, J, and E, represent the December Solstice (January, February, November and December), June Solstice (May, June, July and August), and Equinoxes (March, April, September and October), respectively. Figure 4 indicates that, overall, the average of time lags in Lloyd J are larger than the other two, while the ones in Lloyd D season are opposite. However, the standard deviations in each month are so large that it is difficult to find any significant seasonal differences. Thus, the time lag in large-scale ionospheric responses to solar irradiation does not depend on season.

From longitude differences in the CODE dataset shown in Table 2 indicate that they range from $15^{\circ}$ to $55^{\circ}$. Thus, the corresponding time lags range from $1 \mathrm{~h}$ to $3.7 \mathrm{~h}$. Some studies have already revealed that ionospheric variations are actually most correlated with the solar irradiation in the previous hours, i.e., there are time lags (Chen et al. 2015). Afraimovich et al. (2006) stated that theory predicted a time lag of around $1 \mathrm{~h}$ for global mean TEC variations in response to variations in solar irradiation. A 1 -h time lag corresponds to a $15^{\circ}$ longitude difference. Hocke (2008) found that the time lag between oscillations of solar extreme ultraviolet and global mean TEC were less than $3.5 \mathrm{~h}$, which corresponds to a $52.5^{\circ}$ 

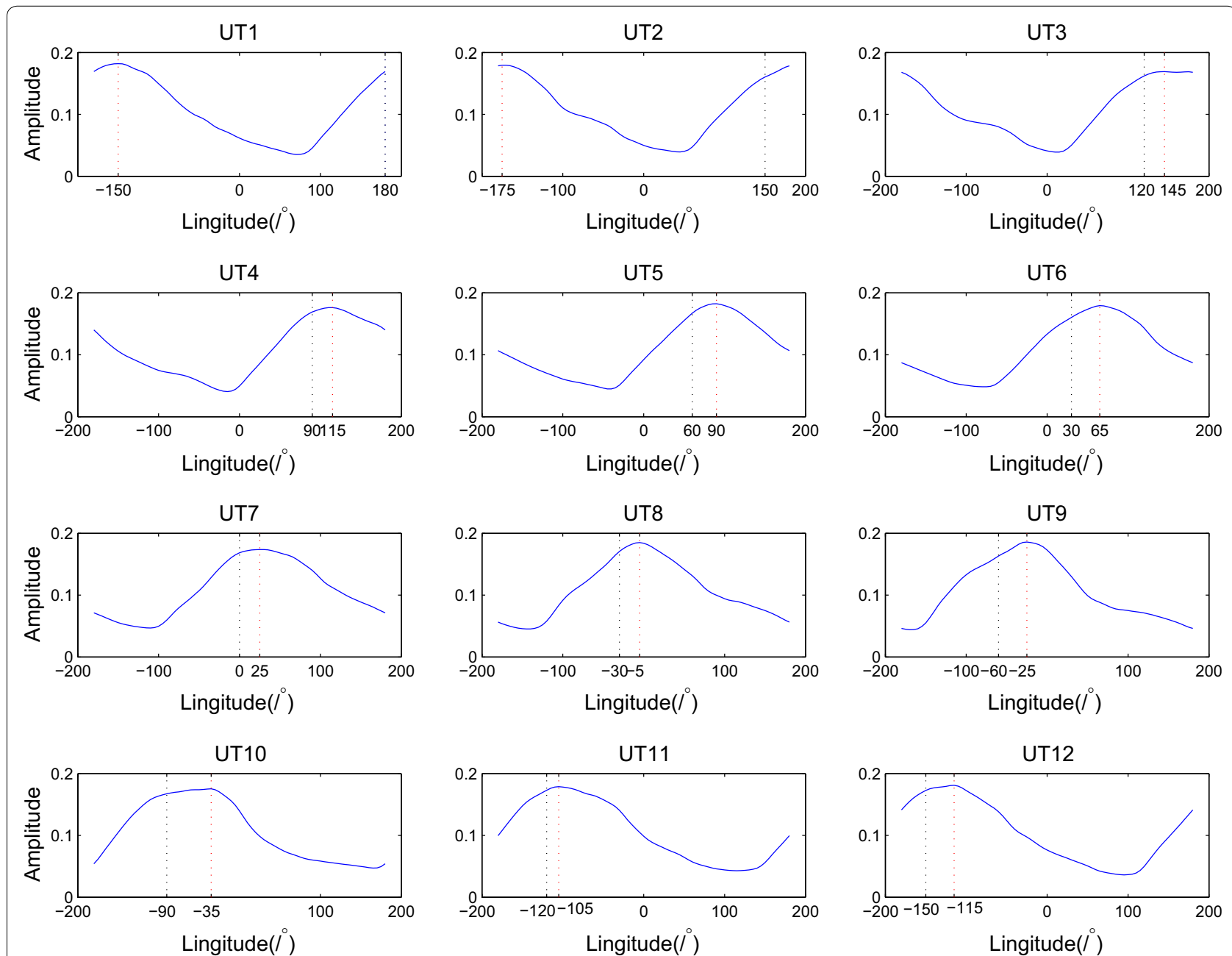

Fig. 3 The large-scale longitudinal variation in the ionosphere for the 12 UTs. The red dotted line indicates the longitude position of the maximum $U^{(2)}$. The black dotted line indicates the longitude position of the maximum solar irradiation. The $x$-axis is the geographic longitude, ranging from $-180^{\circ}$ to $180^{\circ}$

longitude difference (Hocke 2008). While most longitude differences are below $52.5^{\circ}$ in Table 2 , we find there is a $55^{\circ}$ longitude difference, bigger than $52.5^{\circ}$. The difference may be due to the selection of a different dataset. Hocke (2008) selected the oscillations in solar extreme ultraviolet to represent solar radiation.

Before solar irradiation reaches the ionosphere, it should pass the magnetosphere. As the charged particles travel along geomagnetic field lines, the geomagnetic field connects the ionosphere and magnetosphere. The charged particles can travel from the magnetotail to the ionosphere. Conversely, the ionospheric charged particles can travel to the magnetosphere. Concurrently, the geomagnetic field can help propagate plasma waves. The field-aligned currents (FACs) constitute the energetic link between the magnetosphere and ionosphere. Thus, variations in the magnetosphere and ionosphere are coupled and influence each other. Moreover, the thermosphere is the ionosphere background. We speculate that the magnetosphere-ionosphere-thermosphere (M-I-T) system likely results in a time lag.

When solar energy is imported into the M-I-T system, complex processes, such as Joule heating and convection and reconnection between the magnetosphere and ionosphere, likely attribute to the time lags. The response of the ionosphere to variations in ultraviolet radiation flux is determined by the time constants from ionization and the recombination of $\sim 1 \mathrm{~h}$ (Afraimovich et al. 2006; Astafyeva et al. 2008). The ionospheric response has a time delay of $1 \sim 2$ hours to the ring current variation in the magnetosphere, because of the relaxation process in the field-aligned current (Shen 1987). Fung 


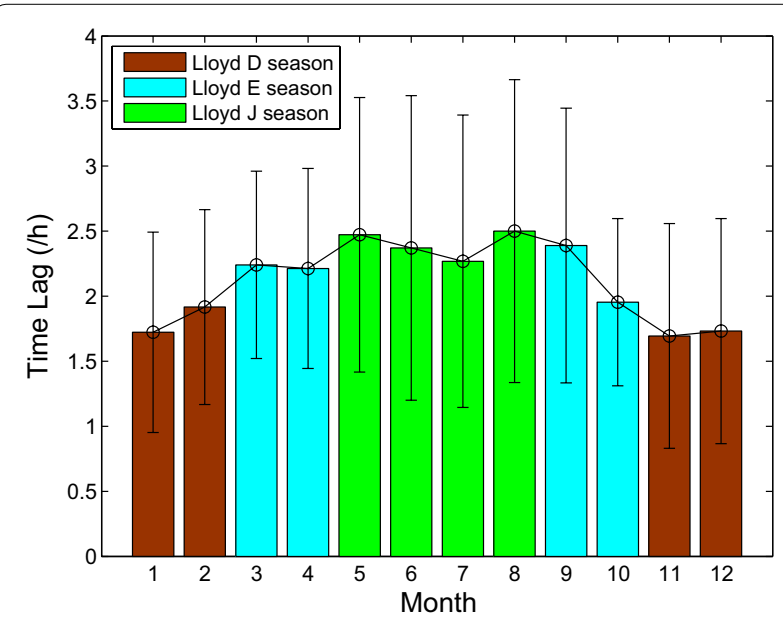

Fig. 4 The averages and standard deviations of the time lags averaged over all UT intervals from 2011-2013 in each month. The vertical bars represent the corresponding standard deviations

and Shao (2008) suggested that the magnetosphere might have a memory time of a few hours, which is caused by a sequence of transitions and configuration changes associated with energy storing, dissipation, and recovery in the magnetosphere. Ionospheric electron density depends on both the ionizing solar radiation and background thermosphere. Thus, some studies attributed ionospheric time lags to thermospheric lags to the solar irradiance (Min et al. 2009; Wang et al. 2006). The correlation between the reversed $D s t$ index and 3-h smoothing Joule heating indicates a delay time of $2-3 \mathrm{~h}$ between the Joule heating and global thermospheric response $(\mathrm{Lu}$ et al. 2017). Hence, we conjecture that the difference in the time lags may indicate different interactions between processes creating large-scale ionospheric variations in the $\mathrm{M}-\mathrm{I}-\mathrm{T}$ system. When the time lag is close to $1 \mathrm{~h}$, ionization and recombination process may play a significant role in the $\mathrm{M}-\mathrm{I}-\mathrm{T}$ system. When the time lag is $2-3 \mathrm{~h}$, Joule heating may be the primary process. Our results provide evidence for the time lag between the maxima of large-scale ionospheric variations and solar irradiation, though we cannot provide an exact explanation for its mechanism due to observational limitations.

\section{Hemispheric asymmetry in geomagnetic coordinates}

Although many research studies on the ionosphere have been conducted based on geographic coordinates (Su et al. 2014; Chen et al. 2015; Talaat and Zhu 2016), some ionospheric characteristics, especially north-south asymmetry, are also investigated compared with geomagnetic coordinates (Geonhwa et al. 2004, 2005; Weihua and Jiaping 2015). Many geospace phenomena, including ionospheric currents, are highly organized by the
Earth's main geomagnetic field. This organization occurs because charged particles can move spirally forward along the magnetic field lines, but not across them. To provide better insight on NSA for ionospheric large-scale characteristics, we convert from geographic to geomagnetic coordinates.

To work in a reference frame that takes the geometry of the geomagnetic field into account, many geomagnetic coordinate systems are designed for different purposes and regions (Laundal and Richmond 2016). For the ionosphere, centered dipole coordinates, which represent a shift of the poles from the rotational axis to the dipole axis, are often used. However, at ionospheric altitude, the geomagnetic field deviates significantly from a centered dipole. To achieve better accuracy with respect to the geomagnetic field, we select the geomagnetic coordinates based on the geomagnetic apex (Laundal and Richmond 2016; Laundal and Gjerloev 2014). The geomagnetic apex coordinates are calculated using a Python wrapper available at GitHub. ${ }^{1}$ In this subsection, we mainly focus on the NSA for large-scale ionospheric variations based on geomagnetic coordinates. After coordinate conversions, the latitudinal results are shown in Fig. 5a. Using the GIMs displayed in geomagnetic coordinates, we construct new tensors whose dimensions are universal time, geomagnetic longitude, and geomagnetic latitude. To investigate the full-time variation, the tensor is constructed using all global VTEC maps every $2 \mathrm{~h}$ from 2011 to 2013. Through rank-1 decomposition, large-scale ionospheric variations in geomagnetic latitude, $U^{(3)}$, are obtained.

Intuitively, a symmetrical large-scale ionospheric variation between the northern and southern hemisphere should place barycenter of the latitudinal variations on the equator. Thus, in this subsection, we preliminarily select the barycenter of geomagnetic $U^{(3)}$ to investigate the NSA for large-scale ionospheric variations. Weighted by the distribution of mass in space, the position of the barycenter is the average position of all parts of the object. In one dimension, the position of the barycenter is usually calculated by $\hat{x}=\frac{\sum_{i} P_{i} x\left(P_{i}\right)}{\sum_{i} P_{i}}$, where $\hat{x}$ is the position of the barycenter, and $P_{i}$ and $x\left(P_{i}\right)$ are the $i$ th amplitude and its corresponding position, respectively. The results are shown in Fig. 5b.

In Fig. 5b, the position of the barycenter is $1.65^{\circ}$ in the southern geomagnetic hemisphere. As depicted by Jee et al. (2010), the negative differences in GIM and TOPEX/Jason TEC are stronger in the southern hemisphere than in the northern hemisphere. Accordingly, due to the sparsity of GPS stations, VTEC estimates in the southern hemisphere are smaller in the CODE GIMs.

\footnotetext{
${ }^{1}$ https://github.com/cmeeren/apexpy.
} 

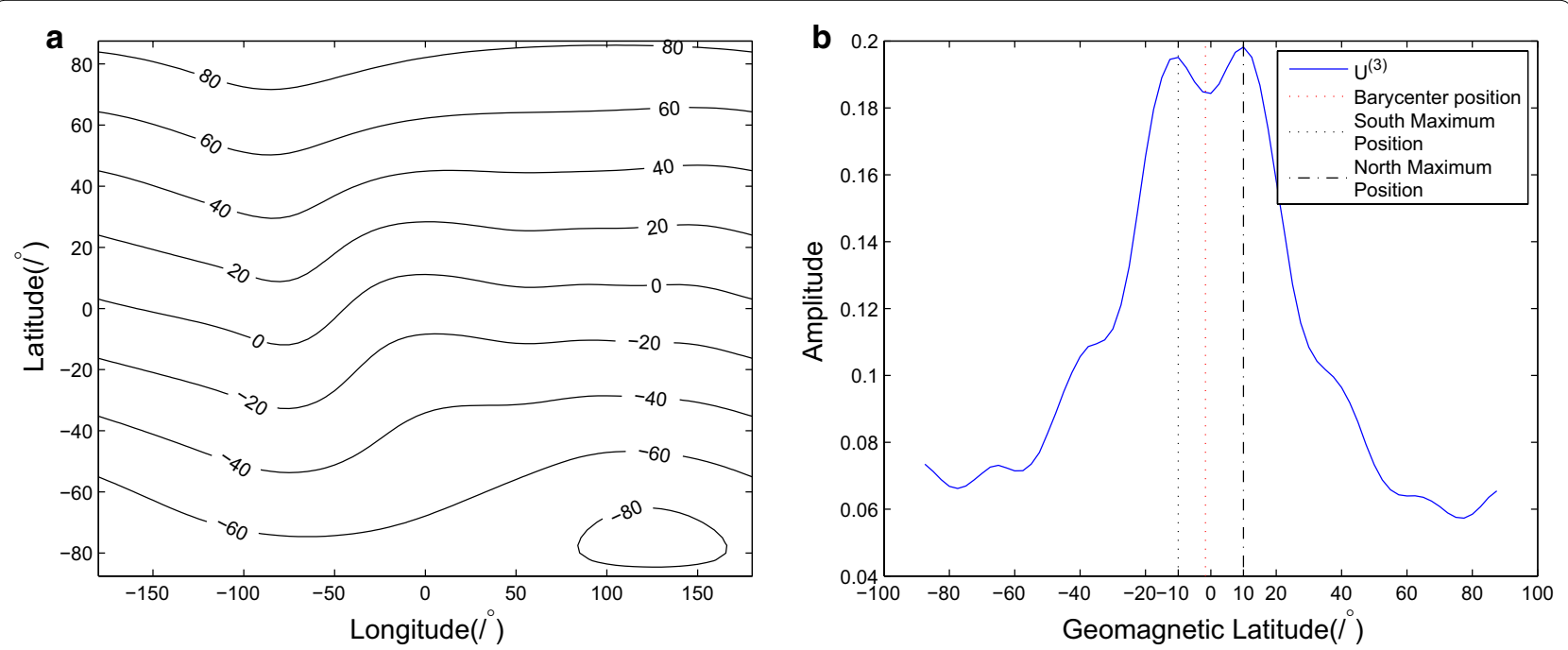

Fig. 5 Large-scale latitudinal variations in the ionosphere in geomagnetic coordinates. a The geomagnetic latitudes based on apex in the geographic coordinate system. $\mathbf{b}$ The latitudinal variation achieved by rank-1 decomposition distributed versus the geomagnetic latitude based on apex. The red, black dotted, and black dotted dash lines indicate the positions of the barycenter, and south and north maximum of $U^{(3)}$, respectively. The $x$-axis is the geomagnetic latitude ranging from $-87.5^{\circ}$ to $87.5^{\circ}$

As Fig. 5b shows, the position of the barycenter is in the southern hemisphere. Because the analysis is made using the CODE VTEC, which is smaller than the real value in the southern hemisphere, we hypothesize that without inhomogeneous coverage of stations, the north-south asymmetry in large-scale ionospheric variations will be clearer. Therefore, an inhomogeneous distribution of GPS stations between the northern and southern hemispheres does not cast doubt on the conclusions regarding NSA for large-scale ionospheric variations acquired from the CODE dataset. The asymmetry shown by the position of the barycenter reveals a north-south hemispheric difference in the large-scale ionospheric variations and provides evidence for NSA based on geomagnetic coordinates. The two crests around the geomagnetic equator indicate the existence of an equatorial ionization anomaly (EIA). As shown in Fig. 5b, the geomagnetic latitude positions of those crests are $10^{\circ} \mathrm{N}$ and $10^{\circ} \mathrm{S}$, showing symmetry. The amplitudes of these two crests are 0.195 and 0.198 , which are almost the same. Therefore, from the perspective of the ionospheric large-scale variation, the asymmetry shown in EIA is not clear.

\section{Daily and diurnal variations of NSA}

We investigate the north-south asymmetry of large-scale ionospheric variations caused by solar ionization referring to geomagnetic coordinates. As introduced above, there are 12 GIMs that constitute a tensor $\mathscr{A}_{12 \times 73 \times 71}$ each day. Here, we use the data displayed in geomagnetic coordinates. Based on the rank-1 decomposition, the geomagnetic latitude mode, $U^{(3)}$ is acquired each day. Then, the barycenter position of $U^{(3)}$ can be calculated to indicate the north-south asymmetry in the large-scale ionospheric variations. Thus, from 2011 to 2013, 1096 barycenter positions $\hat{x}$ are obtained. We investigate the temporal variation in $\hat{x}$ to investigate the solar effect on the NSA. The results are shown in Fig. 6, where its frequency spectrum is calculated by Fourier Transform.

As Fig. 6a shows, the barycenters oscillate seasonally around the geomagnetic equator. The wave form is similar to the trajectory of subsolar position, which oscillates seasonally about the geographic equator. In Fig. 6b, $f_{1}=2.7 \times 10^{-3} \frac{1}{\text { day }}$. The corresponding period is 370 days. There are about 5 days of difference between the daily variation in barycenter position and the subsolar point. Such differences may be attributed to the difference between the geomagnetic and geographic equator, as shown in Fig. 5a. Therefore, such phenomenon can indicate the effect of solar ionization on the NSA for large-scale ionospheric variations.

To compare such NSA between day and night, the CODE dataset displayed in geomagnetic coordinates is divided into two parts, day and night regions. Traditionally, day is defined as 06:00-18:00 LT and night as 18:0006:00 LT. Every day, we select the GIM at UT4(6:00-8:00) when the eastern hemisphere is in day and the western hemisphere is night. Accordingly, from 2011 to 2013, 1096 GIMs at UT4 are selected. The right parts of those GIMs represent day, containing 37 rows of the GIM matrix. Similarly, the left ones represent night, containing 

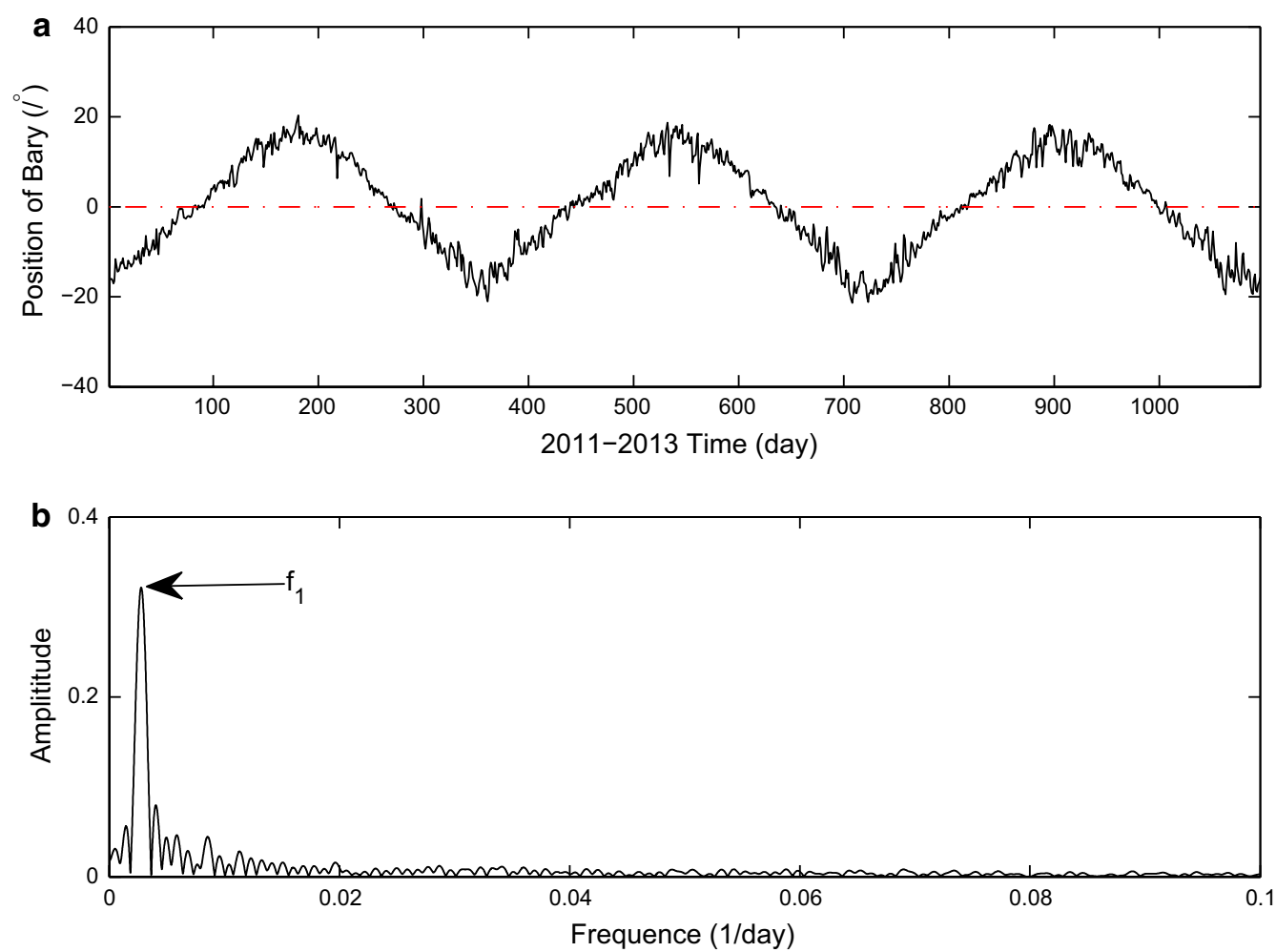

Fig. 6 The daily variation in the barycenter positions of $U^{(3)}$ from 2011 to 2013. a Temporal variations. b The frequency spectra calculated by Fourier Transform

36 rows. Thus, the right parts of the GIMs constitute the day tensor, $\mathscr{A}_{1096 \times 37 \times 71}^{d}$, while the left parts constitute the night tensor, $\mathscr{A}_{1096 \times 36 \times 71}^{n}$. Using the rank-1 decomposition, large-scale ionospheric variations in geomagnetic latitude for day and night are shown in Fig. 7.

As shown in Fig. 7, the barycenter positions of diurnal $U^{(3)}$ are $6.3^{\circ} \mathrm{S}$ and $7.3^{\circ} \mathrm{S}$, which are all in the southern hemisphere. Such phenomenon also provides evidence for north-south asymmetry in large-scale ionospheric variations during day and night. Moreover, both the waveforms of geomagnetic $U^{(3)}$ during the day and night indicate the existence of an EIA. The geomagnetic latitudes of the crests are $10^{\circ} \mathrm{N}$ and $10^{\circ} \mathrm{S}$. The results are in agreement with the conclusions provided in Fig. 5. Thus, the NSAs for large-scale variations in the EIA during day and night are almost identical.

\section{Results of geomagnetic quiet GIMs}

To investigate the effects of geomagnetic disturbances and storms on large-scale ionospheric variations, the GIMs obtained during the geomagnetic quiet periods are employed for analysis. First, although the ionospheric temporal variations are affected by geomagnetic disturbances and storms, those influences are negligible in our analysis. This is because geomagnetic disturbances and storms do not have a significant impact on long-term large-scale variations in the ionosphere. Specifically, the duration of geomagnetic disturbances and storms tends to range from a few minutes to tens of hours ( $\mathrm{Xu} 2009)$, which is short-term compared to our 3-year data length. That is, the time scale of the ionospheric variation caused by the geomagnetic disturbance or storm is short-term compared to 3 years, and the tensor rank-1 decomposition only extracts long-term and large-scale ionospheric variations. Hence, the geomagnetic disturbances and storms would make little difference in the long-term ionospheric variations obtained by the tensor rank-1 decomposition.

As an index measuring the intensity of global geomagnetic activity, the planetary 3-hour-range index $K p$ has been widely used in ionospheric and geomagnetic fields. To further study the effects of geomagnetic disturbances and storms on the ionosphere, we select the $K p$ index as the geomagnetic activity index. Introduced by Bartels et al. (1939), $K p$ is the average $K$-index from 13 geomagnetic observatories between $44^{\circ}$ and $60^{\circ}$ northern or southern geomagnetic latitude, with a 3-h interval and value range of $\left\{0_{0}, 0_{+}, 1_{-}, 1_{0}, \cdots 9_{-}, 9_{0}\right\}$. When $K p<4_{0}$, 

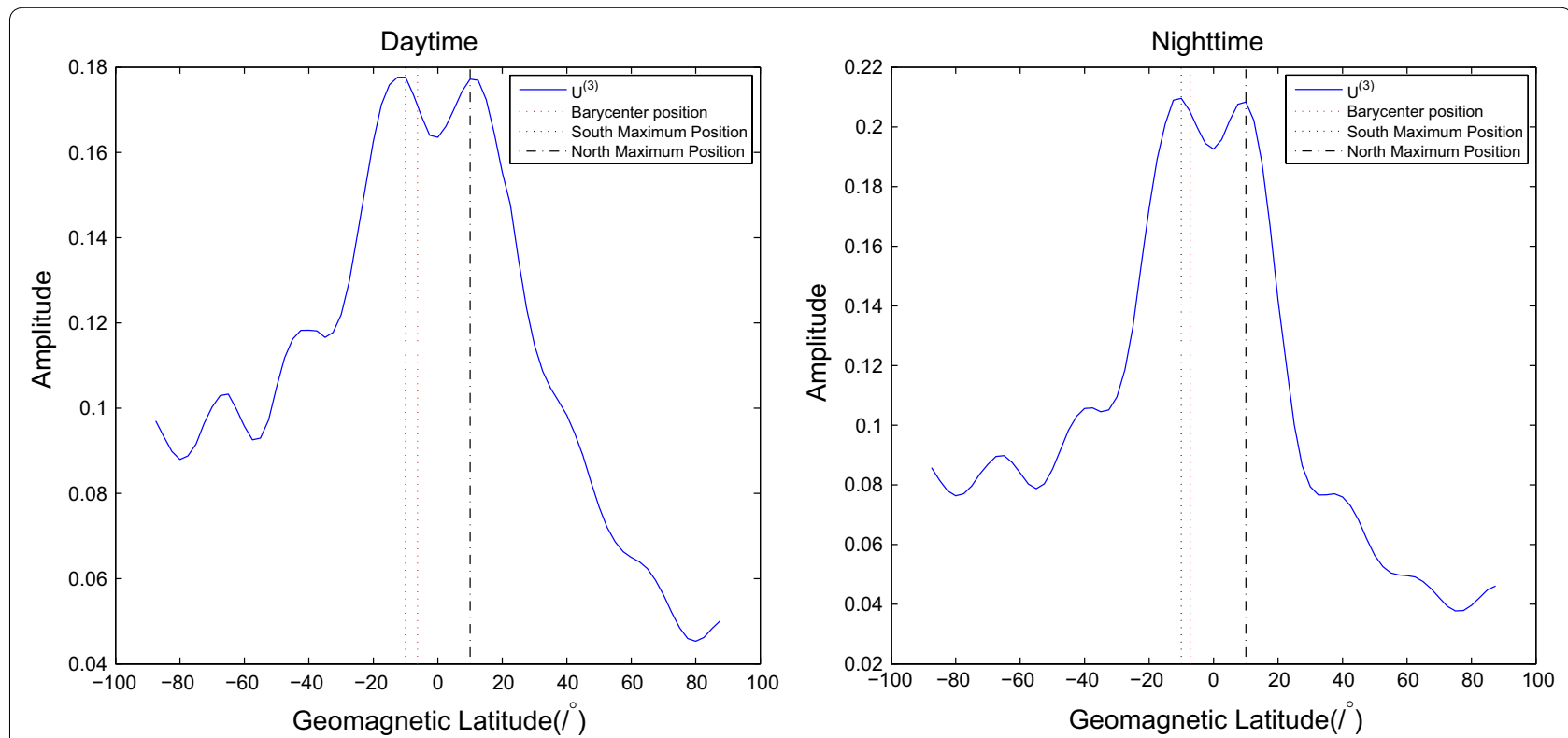

Fig. 7 The diurnal variations in NSA indicated by $U^{(3)}$. The red, black dotted, and black dotted dash lines indicate the positions of the barycenters, and south and north maxima of $U^{(3)}$, respectively. The $x$-axis is the latitude ranging from $-87.5^{\circ}$ to $87.5^{\circ}$. $\mathbf{a}$ Results for daytime. $\mathbf{b}$ Results for night

the geomagnetic field is considered quiet. $K p$ indices are obtained from the National Geophysical Data Center.**2 To study the influences of geomagnetic disturbances and storms on large-scale ionospheric variations, when $K p>4_{0}$, we remove the current GIM and the next one during tensor construction. Hence, the tensor of the ionosphere during geomagnetic quiet periods can be obtained. Then, the tensor rank-1 decomposition is employed to obtain large-scale ionospheric variations during geomagnetic quiet periods.

For large-scale longitudinal patterns via $U^{(2)}$, similar to the previous analysis, we construct a tensor using the geomagnetic quiet GIMs in each UT interval. The number of geomagnetic quiet GIMs in the tensor in each UT interval is shown in the titles of the subfigures in Fig. 8. This figure shows the results of $U^{(2)}$ for geomagnetic quiet GIMs in each UT interval. As shown, the longitude differences between the maxima of large-scale longitudinal variations and those of solar irradiation in $U^{(2)}$ are the same as those in Fig. 3. In addition, removing the GIMs affected by the geomagnetic disturbances and storms, we obtain 11,700 GIMs to investigate the NSA for largescale ionospheric variations. Figure 9 is achieved by tensor rank-1 decomposition during the geomagnetic quiet periods, showing the results of north-south hemispheric asymmetry in large-scale ionospheric variations. The characteristics of $U^{(3)}$ in Fig. 9 are similar with those of $U^{(3)}$ in Fig. 5b. The positions and amplitudes the crests are

\footnotetext{
${ }^{2} \mathrm{ftp}: / /$ ftp.ngdc.noaa.gov/STP/GEOMAGNETIC_DATA/INDICES/KP_AP.
}

$\pm 10^{\circ}$ and about 0.19 . Thus, the NSA in EIA is masked in the large-scale ionospheric variations during the geomagnetic quiet periods. In Fig. 9, the geomagnetic latitude position of the barycenter in $U^{(3)}$ is $1.89^{\circ} \mathrm{S}$. Thus, after removing the influences of geomagnetic disturbances, the results still show the existence of NSA in large-scale ionospheric variations. Therefore, the influences of geomagnetic disturbances and storms make little difference with respect to our previous conclusions. More explicitly, the geomagnetic disturbances/storms have little effect on the results of the tensor rank-1 decomposition in the ionospheric spatiotemporal analysis. The rank-1 decomposition mainly extracts both temporal and spatial variations at large scale in the ionosphere, while the ionospheric variations caused by the geomagnetic disturbances and storms have a relatively small spatiotemporal scale. This phenomenon also shows the robustness of the tensor rank-1 decomposition in analyzing large-scale spatiotemporal variations in the ionosphere.

\section{Conclusions}

As an important component of the geospace environment, the ionosphere has received increasing attention. Research on the ionosphere is of great value in both application and theory. In this paper, VTEC data for 2011-2013 supplied by CODE are employed. The data, which is in the form of a time series of global ionospheric maps, constitute 3D tensors. We propose using the tensor rank decomposition, which can be used to analyze different components in a multidimensional array, to 

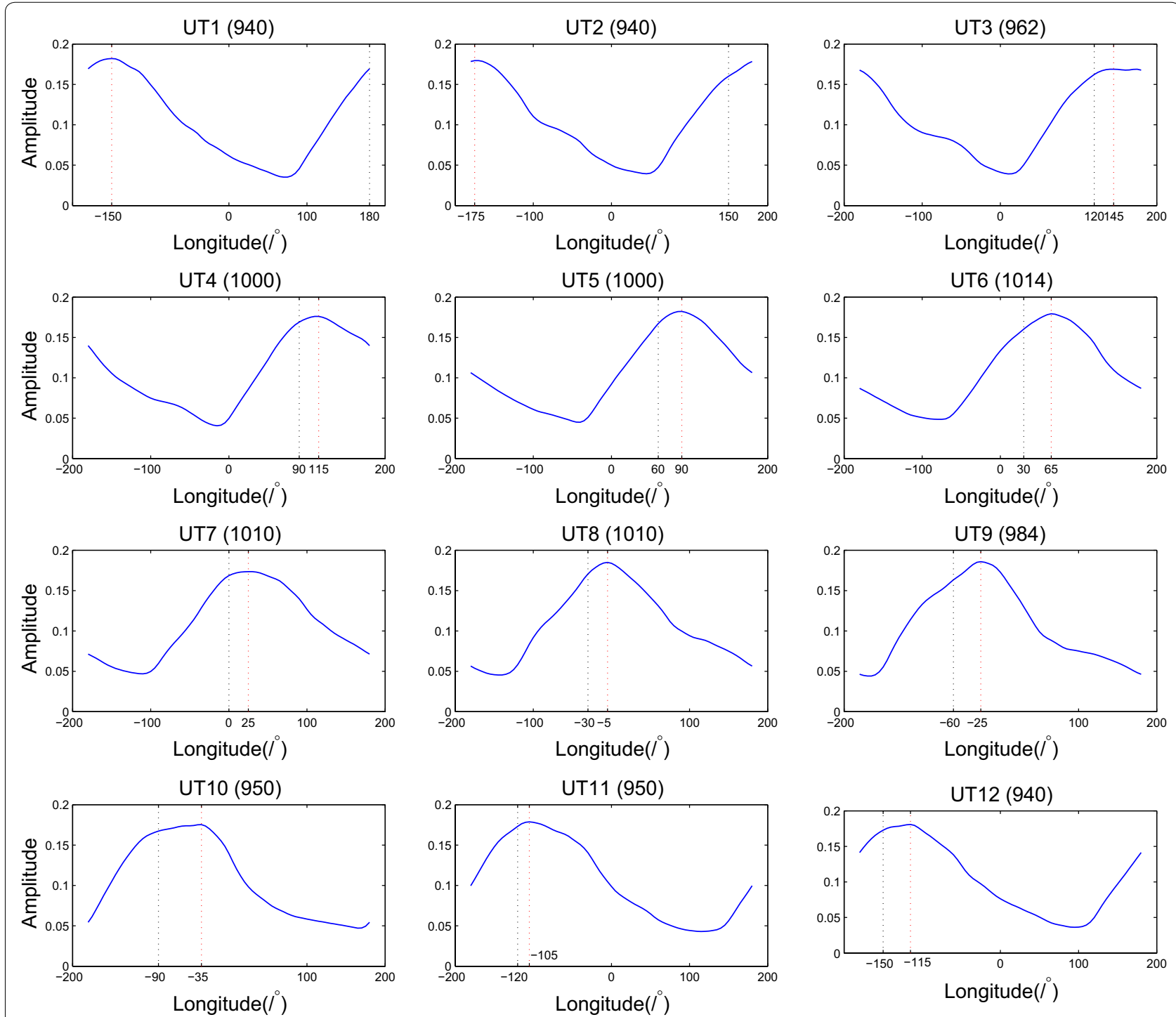

Fig. 8 Large-scale longitudinal variations in the ionosphere for the 12 UTs during the geomagnetic quiet time. The red dotted line indicates the longitude position of the maximum $U^{(2)}$. The black dotted line indicates the longitude position of the maximum solar irradiation. The $x$-axis is the geographic longitude ranging from $-180^{\circ}$ to $180^{\circ}$

jointly analyze the temporal-longitudinal-latitudinal characteristics of the ionosphere. Compared with EOF and PCA, tensor rank decomposition captures both the spatiotemporal and longitudinal-latitudinal characteristics. To analyze the large-scale ionospheric structures, the tensor rank-1 decomposition, also known as the best rank-1 approximation, is used to extract the first principal component of the GIM series.

Through rank-1 decomposition, large-scale ionospheric variations $U^{(1)}, U^{(2)}$ and $U^{(3)}$ are achieved, which are the resulting unit-norm vectors for time, longitude, and latitude mode, respectively. The correspondence between the spectrum of $U^{(1)}$ and solar variation indicates that the rank-1 decomposition describes the large-scale ionospheric temporal variations, mainly caused by solar irradiation. Second, using the GIM tensors for different UT intervals, between the maxima of ionospheric $U^{(2)}$ and solar irradiation, the time lags can be obtained. The time lag primarily ranges from 1 to $3.7 \mathrm{~h}$. Monthly variations in time lags indicates that the time lag between the ionospheric responses and solar irradiation varies without seasonal dependence. The differences in time lags likely indicate different processes in the $\mathrm{M}-\mathrm{I}-\mathrm{T}$ system. When the time lag is close to $1 \mathrm{~h}$, ionization and recombination processes play a significant role in the $\mathrm{M}-\mathrm{I}-\mathrm{T}$ system, while 2-3-h time lags indicate that Joule heating probably 


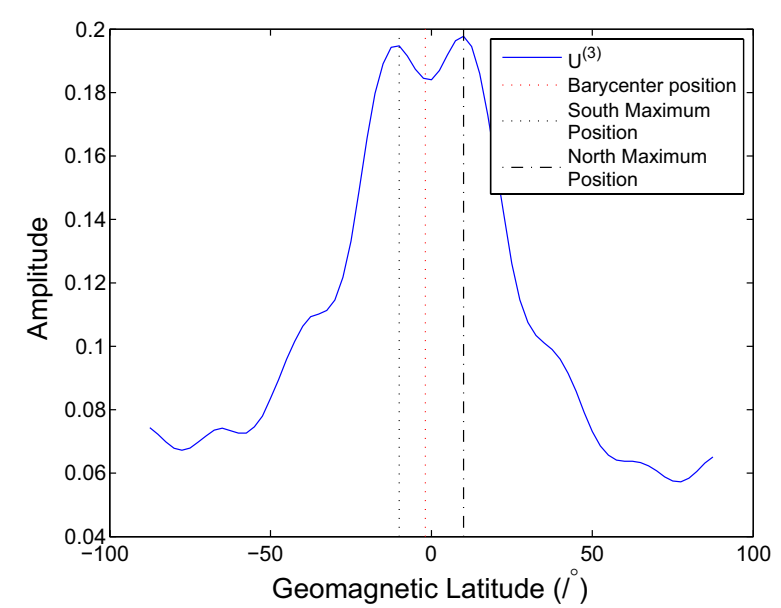

Fig. 9 Large-scale latitudinal variations in the ionosphere in geomagnetic coordinates during the geomagnetic quiet time. The red, black dotted, and black dotted dash lines indicate the positions of the barycenter, and south and north maximum of $U^{(3)}$, respectively. The $x$-axis is the geomagnetic latitude ranging from $-87.5^{\circ}$ to $87.5^{\circ}$

is primary. Moreover, the spatial variation in the rank-1 decomposition is oriented in geomagnetic coordinates. The position of the barycenter for $U^{(3)}$ in geomagnetic coordinates provides evidence for an NSA for large-scale ionospheric variations. Furthermore, the daily variation in hemispheric asymmetry indicates the effect of the Sun on large-scale ionospheric characteristics. The diurnal $U^{(3)}$ in geomagnetic coordinates show that the large-scale variations in the EIA during the day and night are almost identical. Finally, we investigate the influence of geomagnetic disturbances by excluding the GIMs obtained during the geomagnetic disturbances. The results show that the geomagnetic disturbance makes little difference with respect to our primary conclusions. Generally speaking, the rank-1 decomposition can decouple the correlations between time, longitude, and latitude in the ionosphere, and capture smooth ionospheric structures caused by the Sun, geomagnetic field, and magnetosphere.

\section{Authors' contributions}

$\mathrm{SL}$ and $\mathrm{HZ}$ performed the major theoretical analysis and led the writing of the manuscript. SL analyzed the data. XL, YL, and DL helped conduct the research and provided constructive edits to the manuscript. CN and XY participated in the manuscript discussion. All authors read and approved the final manuscript.

\section{Author details}

${ }^{1}$ Department of Electronic Engineering, Tsinghua University, Beijing 100084, China. ${ }^{2}$ Xi'an Research Institute of Hi-Tech, Xi'an 710025, China.

\section{Acknowledgements}

This work was supported by the National Natural Science Foundation of China (41374154 and 41774156). We are grateful to Adam Woods from CIRES, University of Colorado, the David Skaggs Research Center, and Rolf Dach and Stefan Schaer from the Astronomical Institute at the University of Bern. Their help in providing data is appreciated. The GIMs from CODE are available at ftp://ftp.unibe.ch/aiub/CODE. Furthermore, we are grateful to K. M. Laundal from the Birkeland Centre for Space Science, University of Bergen. He kindly helped obtain the geomagnetic coordinates based on apex. We also want to express our thanks to the reviewers for their thoughtful comments and suggestions. Moreover, Shikun Lu would like to thank, in particular, the ongoing and unwavering support from Taotao Sun over the years.

\section{Competing interests}

The authors declare that they have no competing interests.

Ethics approval and consent to participate

Not applicable.

\section{Publisher's Note}

Springer Nature remains neutral with regard to jurisdictional claims in published maps and institutional affiliations.

Received: 17 October 2017 Accepted: 23 February 2018

Published online: 14 March 2018

\section{References}

Afraimovich EL, Astafyeva El, Oinats AV, Yasukevich YV, Zhivetiev IV (2008) Global electron content: a new conception to track solar activity. Ann Geophys 26:335-344

Afraimovich EL, Astafyeva El, Zhivetiev IV (2006) Solar activity and global electron content. Dokl Earth Sci 409(2):921-924

Araujo-Pradere EA, Fuller-Rowell TJ, Codrescu MV, Bilitza D (2005) Characteristics of the ionospheric variability as a function of season, latitude, local time, and geomagnetic activity. Radio Sci 40(5):4629-4629

Astafyeva El, Afraimovich EL, Oinats AV, Yasukevich YV, Zhivetiev IV (2008) Dynamics of global electron content in 1998-2005 derived from global GPS data and IRI modeling. Adv Space Res 42(4):763-769

Atac T, Özgüç A, Rybák J (2005) Overview of the flare index during the maximum phase of the solar cycle 23. Adv Space Res 35(3):400-405

Bartels J, Heck NH, Johnston HF (1939) The three-hour-range index measuring geomagnetic activity. J Geophys Res 44(4):411-454

Chen Y, Liu L, Le H, Zhang H (2015) Discrepant responses of the global electron content to the solar cycle and solar rotation variations of EUV irradiance. Earth Planets and Space 67(80):1-8

Chen Z, Zhang S-R, Coster AJ, Fang G (2015) EOF analysis and modeling of GPS TEC climatology over North America. J Geophys Res Space Phys 120(4):3118-3129

Coyne TNR, Belrose JS (1972) The diurnal and seasonal variation of electron densities in the mid-latitude D region under quiet conditions. Radio Sci $7(1): 163-174$

Ercha A, Huang W, Yu S, Liu S, Shi L, Gong J, Chen Y, Hua S (2015) A regional ionospheric TEC mapping technique over China and adjacent areas on the basis of data assimilation. J Geophys Res Space Phys 120(6):1-13

Espig M, Hackbusch W, Litvinenko A, Matthies ea (2014) Efficient low-rank approximation of the stochastic Galerkin matrix in tensor formats. Comput Math Appl 67(4):818-829

Filisbino TA, Giraldi GA, Thomaz CE (2013) Ranking methods for tensor components analysis and their application to face images. In: 26th Conference on graphics, patterns and images, pp 312-319

Franke S, Yeh K, Andreeva E, Kunitsyn V (2003) A study of the equatorial anomaly ionosphere using tomographic images. Radio Sci 38(1):11-1-11-12

Fung SF, Shao X (2008) Specification of multiple geomagnetic responses to variable solar wind and IMF input. Ann Geophys 26(3):639-652

Geonhwa J, Schunk RW, Ludger S (2004) Analysis of TEC data from the TOPEX/Poseidon mission. J Geophys Res Atmos 109(A1). https://doi. org/10.1029/2003JA010058

Geonhwa J, Schunk RW, Scherliess L (2005) Comparison of IRI-2001 with TOPEX TEC measurements. J Atmos Solar Terr Phys 67(4):365-380

Goldberg R (1966) A theoretical model for the magnetic declination effect in the ionospheric F region. Ann Geophys 22:588-597 
Grasedyck L, Kressner D, Tobler C (2013) A literature survey of low-rank tensor approximation techniques. GAMM-Mitteilungen 36(1):53-78

Guo J, Li W, Liu X, Kong Q (2015) Temporal-spatial variation of global GPS derived total electron content 1999-2013. PLoS One 10(7):1-21

Hanson WB, Moffett RJ (1966) Ionization transport effects in the equatorial $f$ region. J Geophys Res 71(23):5559-5572

Hernández-Pajares M, Juan JM, Sanz J, Orus R, Garcia-Rigo A, Feltens J, Komjathy A, Schaer SC, Krankowski A (2009) The IGS VTEC maps: a reliable source of ionospheric information since 1998. J Geodesy 83(3):263-275

Hocke K (2008) Oscillations of global mean TEC. J Geophys Res 113:4032

Hu J, Ai H, Xue C, He X, Li W, Li H, Xia W, Xie J (2016) lonospheric decontamination based on sparse reconstruction for skywave radar. EURASIP J Adv Signal Process 2016(1):93

Jee G, Lee HB, Kim YH, Chung JK, Cho J (2010) Assessment of GPS global ionosphere maps (GIM) by comparison between CODE GIM and TOPEX/ Jason TEC data: Ionospheric perspective. J Geophys Res Space Phys 115(A10):161-168

Khoromskaia V, Khoromskij BN (2014) Møller-Plesset (MP2) energy correction using tensor factorization of the grid-based two-electron integrals. Comput Phys Commun 185(1):2-10

Kil H, Paxton $\sqcup$ (2011) Causal link of longitudinal plasma density structure to vertical plasma drift and atmospheric tides—a review. Springer, Netherlands

Kolda TG, Bader BW (2009) Tensor decompositions and applications. SIAM Rev 51(3):455-500

Krivova NA, Solanki SK (2002) The 1.3-year and 156-day periodicities in sunspot data: Wavelet analysis suggests a common origin. Astron Astrophys 394(2):701-706

Lathauwer LD, Moor BD, Vandewalle J (2000) On the best rank-1 and rank-(r1, $\mathrm{r} 2$, , r $\mathrm{r}$ ) approximation of higher-order tensor. SIAM J Matrix Anal Appl 21(4):1324-1342

Laundal KM, Gjerloev JW (2014) What is the appropriate coordinate system for magnetometer data when analyzing ionospheric currents? J Geophys Res Space Phys 119(10):8637-8647

Laundal KM, Richmond AD (2016) Magnetic coordinate systems. Space Sci Rev 2017(206):27-59

Lean JL, Meier RR, Picone JM, Sassi F, Emmert JT, Richards PG (2016) Ionospheric total electron content: spatial patterns of variability. J Geophys Res Space Phys 121:10367-10402

Lee WK, Kil H, Kwak Y-S, Wu Q, Cho S, Park JU (2011) The winter anomaly in the middle-latitude $\mathrm{F}$ region during the solar minimum period observed by the constellation observing system for meteorology, ionosphere, and climate. J Geophys Res Space Phys 116:02302

Liu C, Li Y, Pirjola R (2013) Observations and modeling of GIC in the chinese large-scale high-voltage power networks. J Space Weather Space Clim 4:03

Liu I, Zhao B, Wan W, Ning B, Zhang M, He M (2009) Seasonal variations of the ionospheric electron densities retrieved from constellation observing system for meteorology, ionosphere, and climate mission radio occultation measurements. J Geophys Res 114:02302

Lu G, Chappell CR, Schunk RW, Banks PM, Burch JL, Thorne RM (2017) Energetic and dynamic coupling of the magnetosphere-ionosphere-thermosphere system. Wiley, Washington, pp 61-77

Lu H, Plataniotis KN, Venetsanopoulos AN (2006) Multilinear principal component analysis of tensor objects for recognition. In: International conference on pattern recognition, pp 776-779

Min K, Park J, Kim H, Kim V, Kil H, Lee J, Rentz S, Lühr H, Paxton L (2009) The 27-day modulation of the low-latitude ionosphere during a solar maximum. J Geophys Res Atmos 114(A4):27-32

Moussas X, Polygiannakis JM, Preka-Papadema P, Exarhos G (2005) Solar cycles: a tutorial. Adv Space Res 35(5):725-738

Mu W, Wan W, Ren Z, Xiong J (2010) Correlation between ionospheric longitudinal harmonic components and upper atmospheric tides. Sci Bull 55(35):4037-4045

Polygiannakis J, Preka-Papadema P, Moussas X (2003) On signal-noise decomposition of time-series using the continuous wavelet transform: application to sunspot index. Mon Not R Astron Soc 343(3):725-734

Rama G, Pavan K, Balakrishnaiah G, Raja Obul Reddy K, Arafath S, Siva Kumar Reddy N, Chakradhar Rao T, Lokeswara Reddy T, Reddy RR (2016) Evaluation of clearness and diffuse index at a semi-arid station (Anantapur) using estimated global and diffuse solar radiation. Int J Adv Earth Sci Eng 5(1):347-363

Rao KD, Dutt VBSSI (2014) CODE coefficients based single frequency ionospheric error correction model to improve GPS positional accuracy of the low-latitude regions. IOSR J Electron Commun Eng 9(6):18-21

Ren Z, Wan W, Liu L, Zhao B, Wei Y, Yue X, Heelis RA (2008) Longitudinal variations of electron temperature and total ion density in the sunset equatorial topside ionosphere. Geophys Res Lett 35(5):544-548

Schunk R, Nagy A (2009) lonospheres: physics, plasma physics, and chemistry, 2nd edn. Cambridge University Press, Cambridge

Shen CS (1987) The time delay of the ionospheric response to the ring current variation. Chin J Space Sci 8(1):155-162

Su SY, Wu CL, Liu CH (2014) Correlation between the global occurrences of ionospheric irregularities and deep atmospheric convective clouds in the intertropical convergence zone (ITCZ). Earth Planets Space 66(1):1-8

Talaat ER, Zhu X (2016) Spatial and temporal variation of total electron content as revealed by principal component analysis. Ann Geophys 34:1 109-1117

Tsynkov SV (2009) On sar imaging through the earth's ionosphere. SIAM J Imag Sci 2(1):140-182

Wang X, Eastes R, Weichecki Vergara S, Bailey S, Valladares C, Woods T (2006) On the short-term relationship between solar soft X-ray irradiances and equatorial total electron content (TEC). J Geophys Res 111:10-15

Wang H, Ridley AJ, Zhu J (2015) Theoretical study of zonal differences of electron density at midlatitudes with gitm simulation. J Geophys Res: Space Phys 120(4):2951-2966

Wang H, Zhang K (2017) Longitudinal structure in electron density at midlatitudes: upward-propagating tidal effects. Earth Planets Space 69(1):11

Wei N, Shi C, Zou R (2009) Analysis and assessments of IGS products consistencies. Geomat Inf Sci Wuhan Univ 34(11):1363-1367

Weihua Zhengping, Jiaping Xuejing (2015) Characteristics of ionospheric north-south asymmetry and their relationship with irregularity. Wuhan Univ J Nat Sci 20(3):240-246

Wen D, Yuan Y, Ou J, Huo X, Zhang K (2007) lonospheric temporal and spatial variations during the 18 august 2003 storm over China. Earth Planets Space 59(4):313-317

Wright JW (1962) Diurnal and seasonal changes in structure of the midlatitude quiet ionosphere. J Res Natl Bur Stand D Radio Propag 66D(3):297-312

Xu W (2009) Physics of electrodynamics phenomena of the earth. Press of University of Science and Technology of China, Hefei

Xu J, Li X, Liu J, Jing M (2013) Effects of declination and thermospheric wind on TEC longitude variations in the mid-latitude ionosphere. Chin J Geophys 56(5): 1425-1434

Yao YB, Chen P, Zhang S, Chen JJ (2013) Temporal and spatial variations in ionospheric electron density profiles over South Africa during strong magnetic storms. Nat Haz Earth Syst Sci 13(2):375-384

Yoshikawa A, Itonaga M, Fujita S, Nakata H, Yumoto K (1999) Eigenmode analysis of field line oscillations interacting with the ionosphereatmosphere-solid earth electromagnetic coupled system. J Geophys Res 104(A12):28437-28458

\section{Submit your manuscript to a SpringerOpen ${ }^{\circ}$ journal and benefit from:}

- Convenient online submission

- Rigorous peer review

- Open access: articles freely available online

- High visibility within the field

- Retaining the copyright to your article

Submit your next manuscript at $\boldsymbol{\nabla}$ springeropen.com 